\title{
ÉTUDES SUR L'ABSENCE DE LAVAGE DU BEURRE (1)
}

par

\author{
A. H. WHITE (2), R. R. RIEL (3), D. M. BEATTIE (4)
}

K. N. SMITH (2), W. A. MCGUGAN (3)

Le lavage des grains du beurre au cours de la fabrication est une pratique courante. Il a pour objet d'enlever le babeurre libre et, s'il y a lieu, de refroidir les grains. On considère généralement que l'enlèvement du babeurre améliore la qualité de conservation et supprime les mauvaises saveurs, si l'on utilise de la crème de médiocre qualité. Le lavage à l'eau froide s'impose parfois si les grains sont trop tendres pour se prêter à un malaxage normal et il aide aussi à empêcher la formation d'un beurre cassant si la matière grasse a un point de fusion élevé. Si l'eau utilisée n'est pas très bonne, cependant, BOUCHARD [2] préconisait en 1907 de ne pas laver le beurre de crème douce.

En 1920, Wyant [16] a fabriqué dans des conditions analogues des barattées de beurre lavé et non lavé, et il affirmait que l'aptitude à la conservation du beurre lavé était un peu meilleure que celle du beurre non lavé. Mais, il ne présentait pas de données pour étayer son affirmation. Des rapports d'expériences faites au Danemark [5] en 1939 indiquaient que la qualité de conservation du beurre non lavé était égale à celle du beurre lavé. JENSEN et ses associés [6] ont confirmé, en 1942, les résultats obtenus au Danemark. En 1945, O'Shea et Lyons [10] ont étudié l'effet de la teneur en caillé sur la qualité de conservation du beurré et ont constaté que le beurre à gros grains $(0,92 \%$ de caillé) avait généralement une meilleure saveur et plus d'arôme après une période d'entreposage que le beurre à petits grains $(0,49 \%$ de caillé). En 1946, Pont [11] a constaté qu'après trois mois de conservation à $16^{\circ} \mathrm{F}$., le beurre lavé était un peu supérieur au beurre non lavé : cette constatation n'a pas été confirmée dans d'autres expériences faites en 1948 [12]. Les conclusions des expériences danoises ont été corroborées par d'autres chercheurs du Canada [1], de l'Australie [7, 9] et de la Nouvelle-Zélande [4, 8].

Quelques-unes des études ci-dessus ont aussi indiqué que lorsqu'on utilisait de la crème de qualité plutôt médiocre, la qualité de conservation des beurres lavés et non lavés était la même. Mais, dans aucune de ces études, on n'a cherché la corrélation entre

(1) Le Québec laitier, 1957, 16, no 3, p. 14). Travail entrepris et conduit par le Ministère fédéral de l'Agrieulture du Canada : (2) Division de la mierobiologie, Service des sciences; (3) Division de la chimie, Service des sciences ; (4) Division des produits laitiers, Service des marchés. 
l'acidité de la erème et la qualité de conservation des beurres lavé et non lavé. En outre, les conditions de fabrication et d'entreposage dans des études faites antérieurement dans d'autres pays n'étaient pas les mêmes qu'au Canada.

En dépit des constatations faites dans les recherches antérieures, un vœu fut adopté au congrès annuel de l'Association des fabricants de beurre de l'Ontario, en décembre 1952, pour demander que l'on étudie davantage le problème. Au début de 1953, réunis à Ottawa, les chefs des Divisions provinciales de l'industrie laitière appuyaient aussi le projet. Par conséquent, avec la pleine collaboration de la Division des produits laitiers du Service des marchés, l'étude dont il est fait état ici a été entreprise en vue de recueillir des données fondées sur des expériences réalisées sous conditions canadiennes de fabrication. Il s'agissait de déterminer l'effet de l'absence de lavage dans la fabrication du beurre sur la saveur de celui-ci avant et après l'entreposage et sur la teneur en caillé. On voulait aussi déterminer l'effet que l'absence du lavage exerce sur les numérations bactériennes et sur quelques-uns des facteurs chimiques qui peuvent modifier la qualité de conservation du beurre.

\section{Conduite de l'expérience}

Vingt-neuf beurreries réparties dans tout le Canada furent choisies par les chefs des divisions provinciales de l'industrie laitière, en vue d'utiliser des crèmes de toutes sortes de qualités et de couvrir autant que possible les variations de la qualité de l'eau. Des barattées de beurre lavé et non lavé provenant de bassins fractionnés de crème ont été fabriquées chaque mois, de juin à novembre 1953 , sous la surveillance des inspecteurs laitiers provinciaux. La normalisation de l'humidité des beurres non lavés a été faite avec du babeurre du barattage. Les beurreries avaient instruction d'ajouter $2 \%$ de sel au beurre. Six échantillons de sept livres ont été emballés de chaque barattée et bien refroidis dans un congélateur rapide. Cinq boîtes ont été envoyées à Toronto pour le classement et l'entreposage, et une à Ottawa pour le classement initial et l'analyse. La plupart des échantillons ont été expédiés sans réfrigération et, sauf pour quelques exceptions survenues pendant la période de chaleur, ils sont arrivés dans un état satisfaisant. A l'arrivée, le beurre a été entreposé à $-5^{0}$. F.

Qualité de la crème. - Pour donner suite au plan qui avait été dressé, il y avait amplement de variation dans l'acidité de la crème d'une fabrique à l'autre, mais, pour une même fabrique, l'acidité était assez uniforme pendant toute la saison. L'acidité de la crème au quai de réception variait de 0,11 à $0,58 \%$. Divers composés sodiques ont été employés pour la neutralisation. L'acidité au 
barattage variait de 0,07 à $0,24 \%$, mais chez $64,7 \%$ des barattées, elle variait de 0,10 à $0,16 \%$.

Classement. - Chaque mois, pendant une période de six mois, un échantillon frais de chaque barattée a été classé. Les échantillons servant au classement initial et à l'échantillonnage ont été remis en entrepôt frigorifique pour une période de dix mois, gardés ensuite pendant deux semaines à $50^{\circ} \mathrm{F}$. pour reproduire les conditions de la distribution au détail et reclassés. Les classements ont été faits par deux classificateurs de produits laitiers expérimentés qui n'avaient aucune connaissance antérieure du type de beurre. La notation a été faite suivant les normes canadiennes de classement [3], sauf que l'on a employé les désignations "plus » $(+)$ et "marginal" (M) pour indiquer de petites différences dans la saveur.

Analyse. - Les méthodes employées pour les déterminations chimiques et microbiologiques ont été exposées dans des publications antérieures $[13,14,15]$.

\section{Résultats et discussion}

Qualité de la saveur. - On n'a pas noté de différence significative dans le pointage de la saveur des beurres lavés et non lavés au classement initial, ni après l'entreposage. C'est ce qui ressort de la distribution des échantillons d'après la saveur, distribution qui fait l'objet du tableau I. Bien qu'il y ait eu une autre perte dans le pointage de la saveur après dix mois d'entreposage à - $5^{\circ} \mathrm{F}$., suivi de deux semaines à $50^{\circ} \mathrm{F}$., le pointage a été le même pour les deux types de beurre. Le pointage de la saveur de la plupart des échantillons dont le total initial était de 40 et $39+$ a diminué après six mois d'entreposage, mais aucun échantillon n'a été ramené à une eatégorie plus basse. D'autre part, $74 \%$ des échantillons dont le pointage initial de la saveur était de 39 et $39 \mathrm{M}$ et $65,5 \%$ de beurres notés à 38 ont gardé leur pointage initial de saveur après six mois d'entreposage. La perte moyenne de notation de saveur pour les beurres lavés et non lavés a été de 0,21 et 0.22 point respectivement, après six mois d'entreposage à $-5^{\circ} \mathrm{F}$. et 0.31 et 0.34 point après dix mois d'entreposage à $-5^{\circ} \mathrm{F}$., suivi de deux semaines à $50^{\circ} \mathrm{F}$. Le pourcentage élevé de barattées comparables qui ont obtenu le même pointage de saveur à tous les classements témoignent de la qualité analogue des beurres lavés et non lavés. Ces chiffres sont présentés au tableau II.

Acidité de la crème. - L'acidité initiale de la crème a eu un. effet déterminé sur la notation de la saveur des beurres après six mois d'entreposage. Pour chaque accroissement de $0,1 \%$ de l'acidité initiale, il y a eu augmentation du pourcentage des beurres 
TABLEAU I

EFFET DE L'ENTREPOSAGE

SUR LA DISTRIBUTION DES ÉCHANTILLONS DE BEURRE

D'APRES LA NOTION DE LA SAVEUR

APRÉS SIX MOIS D'ENTREPOSAGE A $-5^{\circ} \mathrm{F}$

\begin{tabular}{|c|c|c|c|c|c|c|c|c|}
\hline \multirow{2}{*}{$\begin{array}{l}\text { Notation } \\
\text { initiale } \\
\text { de saveur }\end{array}$} & \multirow{2}{*}{$\begin{array}{l}\text { Nombre } \\
\text { de } \\
\text { barattées }\end{array}$} & \multicolumn{7}{|c|}{ Notation de la saveur après l'entreposage } \\
\hline & & 40 & $39+$ & 39 & $39 \mathrm{Ma}$ & 38 & 37 & 36 \\
\hline
\end{tabular}

(Nombre de barattées)

BEURRE LAVÉ

\begin{tabular}{l|r|l|l|l|l|l|l|l}
40 & 8 & 1 & 2 & 5 & - & - & - & - \\
$39+$ & 17 & - & 3 & 13 & 1 & - & - & - \\
39 & 98 & - & 1 & 72 & 20 & 5 & - & - \\
$39 \mathrm{Ma}$ & 33 & - & - & 15 & 10 & 6 & 1 & 1 \\
38 & 14 & - & - & - & - & 9 & 3 & 2 \\
Total & 170 & 1 & 6 & 105 & 31 & 20 & 4 & 3 \\
$\%$ & - & 0.6 & 3.5 & 61.8 & 18.2 & 11.8 & 2.4 & 1.7
\end{tabular}

BEURRE NON LAVÉ

\begin{tabular}{l|r|r|l|l|l|l|l|l}
40 & 8 & - & 3 & 4 & 1 & - & - & - \\
$39+$ & 15 & - & 1 & 13 & 1 & - & - & - \\
39 & 90 & - & 1 & 67 & 18 & 4 & - & - \\
$39 \mathrm{Ma}$ & 42 & - & - & 12 & 17 & 11 & 1 & 1 \\
38 & 15 & - & - & - & 1 & 9 & 3 & 2 \\
Total & 170 & - & 5 & 96 & 38 & 24 & 4 & 3 \\
$\%$ & - & - & 2.9 & 56.5 & 22.4 & 14.1 & 2.4 & 1.7 \\
\hline
\end{tabular}

$\mathbf{M}_{\mathfrak{x}}=$ marginal

TABLEAU II

SOMMAIRE DE BARATTÉES GOMPARABLES

AYANT LES MEMES NOTATIONS DE SAVEUR ET DES NOTATIONS DIFFERENTES

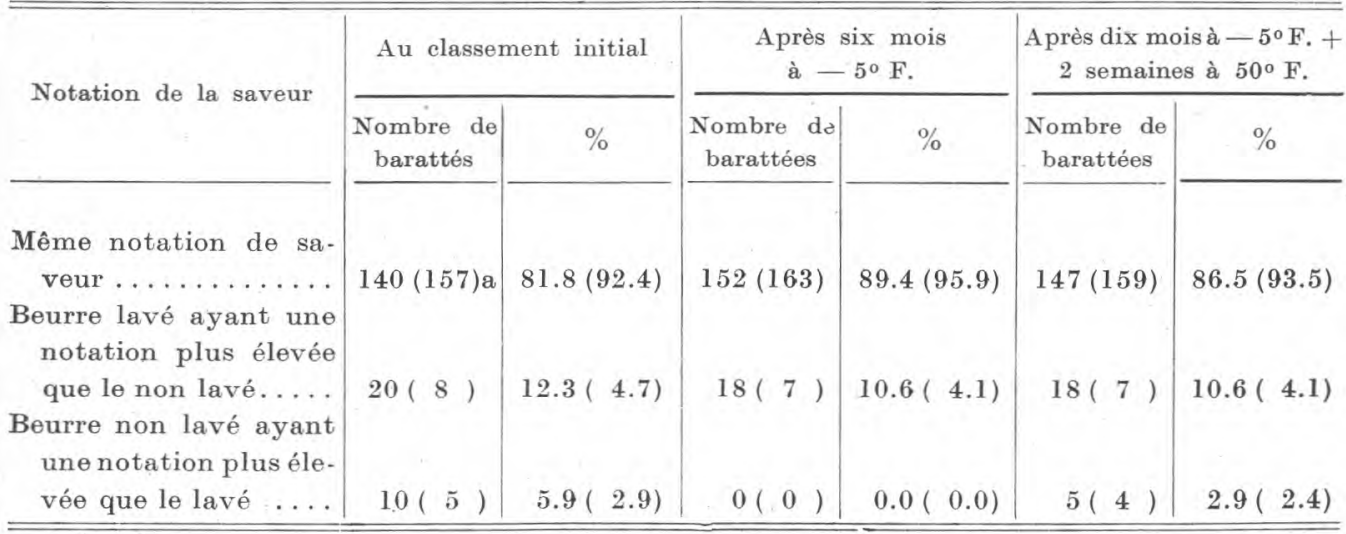

Les chiffres entre parenthèses sont les résultats obtenus lorsqu'on a considéré les beurres ayant des notations de 39 et de $39 \mathrm{M}$ (marginal) comme étant de même qualité. 
dont la notation de la saveur a baissé. Le pourcentage des échantillons dont la notation de saveur a été abaissée a été le même pour le beurre lavé et le beurre non lavé. Après dix mois d'entreposage à $-5^{\circ} \mathrm{F}$., suivi de deux semaines à $50^{\circ} \mathrm{F}$., le pourcentage d'échantillons dont le pointage de saveur a été abaissé était le même pour chaque intervalle de variation de l'acidité de la crème.

Cette étude a établi que sous les conditions canadiennes de fabrication commerciale, avec une variété étendue de qualités de crème, la qualité de la saveur, au début et après l'entreposage, était aussi bonne chez les beurres non lavés que chez les beurres lavés correspondants. Ces résultats concordent avec ceux des chercheurs du Danemark, de l'Australie, de la Nouvelle-Zélande et du Canada.

Qu'il n'y ait pas eu de différence significative dans la qualité de la saveur entre les beurres lavé et non lavé, cela peut tenir en grande partie aux méthodes modernes de production, à la neutralisation et à la pasteurisation de la crème au cours de la fabrication, et à l'emploi d'un matériel en acier inoxydable et d'entrepôts frigorifiques modernes.

Teneur en caillé. - L'effet de l'absence de lavage sur la teneur du beurre en caillé fait l'objet du tableau III. La teneur moyenne en caillé du beurre non lavé a dépassé de $0,53 \%$ celle du beurre lavé. Cependant, il y a eu des variations très amples entre la teneur en caillé de couples individuels de barattées de beurre lavé et non lavé et d'une beurrerie à l'autre. Ces écarts ont varié de 0,15 à $0,85 \%$ entre les couples de barattées et de 0,35 à $0,77 \%$ entre les beurreries.

TABLEAU III

TENEUR EN GAILlE DE BEURRES LAVE ET NON LAVÉ

\begin{tabular}{|c|c|c|c|c|c|}
\hline & \multicolumn{2}{|r|}{ Lavé } & \multicolumn{2}{|c|}{ Non lavé } & \multirow{2}{*}{$\begin{array}{c}\text { Ecart } \\
\text { à la } \\
\text { moyenne } \\
\%\end{array}$} \\
\hline & $\begin{array}{c}\text { Moyenne } \\
\%\end{array}$ & $\begin{array}{c}\text { Extrêmes } \\
\%\end{array}$ & $\begin{array}{c}\text { Moyenne } \\
\%\end{array}$ & $\begin{array}{c}\text { Extrêmes } \\
\%\end{array}$ & \\
\hline $\begin{array}{l}\text { Tous les beurres ...... } \\
\text { Beurreries individuelles } \\
\text { (moyenne de 6 barat - } \\
\text { tées) }\end{array}$ & 0.78 & $0.42-1.24$ & 1.31 & $0.93-1.69$ & 0.53 \\
\hline Minimum $\ldots . .$. & 0.51 & $0.45-0.61$ & 1.09 & $0.93-1.29$ & 0.35 \\
\hline Maximum ........ & 1.12 & $0.96-1.24$ & 1.48 & 1. $39-1.56$ & 0.77 \\
\hline
\end{tabular}

La teneur élevée en caillé de quelques beurres lavés est attribuée à ce qu'on limite le lavage à cause d'un approvisionnement d'eau déficient en qualité ou en quantité. La teneur plutôt basse en caillé 
de quelques barattées non lavées est due surtout à la grande dilution de la crème qui est causée par le rinçage des pots (bidons). La teneur en caillé peut aussi avoir été influencée, par la grosseur des grains et la proportion de lait de beurre laissé dans la baratte. L'augmentation de $0,53 \%$ de la teneur en caillé, augmentation due à l'absence de lavage, correspond à celles qui ont été signalées par d'autres chercheurs.

En théorie, si l'on considère un sérum de crème renfermant $9 \%$ d'extrait sec dégraissé et un beurre de 15,9\% d'humidité venant de ce sérum, la teneur en caillé qui est calculée serait la suivante :

$$
\frac{15.9 \times 9.0}{91.0}
$$

La composition moyenne de tous les beurres a été la suivante :

$\begin{array}{lcccc} & \text { Humidité } & \text { Matière grasse } & \text { Sel } & \text { Saillé } \\ \text { Non lavés .... } & \mathbf{1 5 . 4 9 \%} & 81.20 \% & 2.01 \% & 1.31 \% \\ \text { Lavés ...... } & \mathbf{1 5 . 6 1 \%} & 81.59 \% & 2.02 \% & 0.78 \%\end{array}$

Facteurs chimiques. - Les valeurs chimiques de la matière grasse qui révèlent les défauts dus à l'oxydation et au rancissement ont été mesurées par des analyses chimiques, avant et après l'entreposage. Ces valeurs chimiques ont été essentiellement les mêmes pour les beurres lavés et non lavés, et elles n'ont pas varié de façon significative au cours de l'entreposage. Il suit que la détérioration de la saveur n'était pas attribuable à une oxydation, ni à une hydrolyse marquée de la matière grasse.

Cuivre et fer. - Un certain nombre de travaux de recherches ont indiqué que la détérioration de la matière grasse par oxydation est causée par le cuivre et le fer. Une teneur en cuivre de $0,15 \%$ o et une teneur en fer de $1,0 \%$ o sont considérées comme les limites maximums pour la bonne conservation de la qualité du beurre fabriqué avec de la crème neutralisée. Sur les 154 échantillons analysés au cours de nos travaux, 12 seulement ont dépassé $0,15 \%{ }^{\circ}$ de cuivre et, $24,1,0 \%$ de fer. Deux échantillons seulement avaient des teneurs en cuivre et en fer supérieures aux deux limites ci-dessus et les deux échantillons avaient, au début et après l'entreposage, une notation de saveur 38 .

La teneur moyenne en cuivre de ces beurres était de $0,07 \%$, et la teneur moyenne en fer, de $0,70 \%$. Le lavage n'a pas exercé d'effet significatif sur la teneur en fer, mais, dans quelques cas, il a abaissé la teneur en cuivre du beurre. Comme le cuivre et le fer sont liés au sérum du beurre, leur teneur dans le beurre lavé peut avoir été affectée par la pureté de l'eau et le degré de lavage. 
$p H$. - La distribution des beurres suivant leur $p H$ est présentée au tableau IV. La plupart d'entre eux, soit 70\% des beurres lavés et $60,5 \%$ des beurres non lavés, avaient des $p H$ situés entre 7,0 et 8,0 . D'une façon générale, les valeurs $p H$ des beurres lavés excédaient de 0,2 unités celles des beurres non lavés. La moyenne des valeurs $p \mathrm{H}$ des beurres lavés était de 7,4 , et celle des beurres non lavés, de 7,2. L'entreposage n'a eu aucun effet sur les valeurs $p H$.

TABLEAU IV

DISTRIBUTION DES BEURRES SUIVANT LES VALEURS pH

\begin{tabular}{|c|c|c|c|c|}
\hline \multirow{2}{*}{ Intervalle d'acidité } & \multicolumn{2}{|c|}{ Lavé } & \multicolumn{2}{|c|}{ Non lavé } \\
\hline & Nombre & $\%$ & Nombre & $\%$ \\
\hline-6.49 & 7 & 4.0 & 19 & 11.3 \\
\hline $6.5-6.99$ & 25 & 14.7 & 32 & 19.8 \\
\hline $7.0-7.49$ & 71 & 41.7 & 64 & 37.6 \\
\hline $7.5-7.99$ & 50 & 29.5 & 39 & 22.9 \\
\hline $8.0-8.49$ & 16 & 9.4 & 15 & 8.8 \\
\hline $9.0-$ & 1 & 0.6 & 1 & 0.6 \\
\hline
\end{tabular}

On n'a pas noté de corrélation nette entre les valeurs $p H$ et la notation de la saveur de ces beurres. Un $p H$ bas (acidité élevée) provoque des défauts de saveur dus à l'oxydation dans le beurre d'entreposage. Cependant, la plupart de ces beurres avaient des valeurs $p H$ supérieures à la limite inférieure de 6,8 qui est préconisée pour une bonne qualité de conservation chez le beurre entreposé.

Numérations microbiologiques. - La distribution des échantillons, d'après les numérations bactériennes, a été la même pour les beurres lavés et non lavés, comme on le voit au tableau V. Les moyennes logarithmiques des numérations bactériennes étaient un peu plus basses pour les beurres lavés que pour les beurres non lavés, tant avant qu'après l'entreposage. Dans la plupart des cas, les numérations ont diminué au cours de l'entreposage. On n'a observé aucune tendance régulière dans les numérations bactériennes de couples individuels de barattées. A l'examen initial, $57,7 \%$ des couples de barattées avaient des numérations bactériennes du même ordre, tandis que 18,2\% des beurres lavés et $24,1 \%$ des beurres non lavés avaient des numérations supérieures à celles de leurs contreparties : on a observé une diminution analogue après l'entreposage. Des différences dans les numérations bactériennes de couples de barattées ont généralement été notées dans les intervalles inférieurs à 50.000 par millilitre. Les moyennes 
TABLEAU $V$

DISTRIBUTION DES BEURRES SUIVANT LA NUMÉRATION BAGTÉRIENNE

\begin{tabular}{|c|c|c|c|c|c|c|c|c|}
\hline \multirow{3}{*}{$\begin{array}{c}\text { Intervalle } \\
\text { des numérations } \\
\text { par millitre }\end{array}$} & \multicolumn{4}{|c|}{ Classement initial } & \multicolumn{4}{|c|}{ Après l'entreposage } \\
\hline & \multicolumn{2}{|c|}{ Lavé } & \multicolumn{2}{|c|}{ Non lavẻ } & \multicolumn{2}{|c|}{ Lavé } & \multicolumn{2}{|c|}{ Non lavé } \\
\hline & Nomb & e $\%$ & Nombre & $\%$ & Nombre & $\%$ & Nombre & $\%$ \\
\hline 5,000 et moins. & 59 & 34.7 & 50 & 31.8 & 80 & 47.6 . & 74 & 43.8 \\
\hline $5,001-10,000$ & 31 & 18.2 & 26 & 15.3 & 30 & 17.9 & 29 & 17.2 \\
\hline $10,001-20,000$ & 24 & 14.1 & 33 & 19.4 & 15 & 8,9 & 25 & 14.8 \\
\hline $20,001-50,000$ & 18 & 10.6 & 20 & 11.8 & 14 & 8.3 & 15 & 8.9 \\
\hline $50,000-100,000$ & 23 & 13.5 & 16 & 9.4 & 11 & 6.5 & 9 & 5.3 \\
\hline $100,001-250,000$ & 11 & 6.5 & 11 & 6.5 & 6 & 3.6 & 6 & 3.5 \\
\hline $250,001-500,000$ & 4 & 2.4 & 5 & 2.9 & 6 & 3.6 & 5 & 3.0 \\
\hline Plus de $500,000 \ldots$ & - & 一 & & 2.9 & & 3.6 & 6 & 3.5 \\
\hline Moyenne $\log \ldots .$. & & 000 & 13 & 000 & 6,5 & 500 & & 100 \\
\hline
\end{tabular}

logarithmiques des numérations bactériennes de couples de barattées fabriquées dans la même beurrerie étaient plus basses pour les beurres lavés chez 18 beurreries, et plus basses pour les beurres non lavés chez 11 beurreries.

Les numérations totales de moisissures et de levures ont été en général très basses. Environ $70 \%$ des beurres lavé et non lavé avaient des numérations inférieures à 50 par millilitre. Avant l'entreposage, $67 \%$ des couples de barattées entraient dans la même catégorie et $12 \%$ des beurres lavés et $21 \%$ des non lavés avaient une numération supérieure à sa contrepartie.

\section{Numérations d'organismes proléolytiques et hipolytiques}

Les organismes protéolytiques et lipolytiques sont importants dans le beurre, à cause de l'effet qu'ils peuvent exercer sur la qualité de la saveur. Les colonies protéolytiques étaient présentes chez tous les beurres. Elles représentaient en moyenne 10\% de la numération bactérienne totale et, chez quelques échantillons de beurres lavés et non lavés accusant généralement des numérations basses, ces groupes d'organismes atteignaient un maximum de $60 \%$ des colonies totales. Une proportion élevée des couples de barattées entrait dans le même intervalle de numération, plus de $70 \%$ des beurres ayant des numérations inférieures à 5.000 par millilitre. On a retrouvé des colonies lipolytiques chez seulement environ $35 \%$ des beurres lors de l'examen initial, les numérations se situant entre moins de 100 et 6.000 par millilitre. Après l'entreposage, seulement 23 beurres lavés et 21 beurres non lavés ont accusé la présence de colonies lipolytiques. 
Organismes coliformes. - A l'examen initial, 10\% seulement des beurres lavés et $15 \%$ des beurres non lavés ont manifesté la présence d'organismes coliformes, et seulement 5 beurres lavés ainsi que 10 non lavés avaient des numérations supérieures à 10 par millilitre. Après l'entreposage, on a trouvé des organismes coliformes chez seulement trois échantillons des beurres lavés et trois des beurres non lavés.

On a observé dans les numérations de quelques barattées individuelles des écarts plutôt marqués qui étaient difficiles à expliquer. Chez les beurreries qui n'avaient qu'une seule baratte, ces écarts peuvent être dus à l'ordre de succession des diverses barattées, puisque la crème de la première barattée recueillerait la majeure partie de la contamination microbienne du matériel. Dans quelques cas, les numérations beaucoup plus élevées des beurres lavés pourraient être attribuées à la contamination par un approvisionnement d'eau à numérations élevées. On n'a trouvé aucune corrélation apparente entre les numérations bactériennes et la notation de la saveur, même après dix mois de conservation à $-5^{\circ} \mathrm{F}$. suivi de deux semaines à $50^{\circ} \mathrm{F}$. Cela tient probablement à l'absence ou à la rareté d'organismes aptes à provoquer une détérioration de la saveur.

Quelques considérations pratiques. - La consistance et la texture des beurres lavés et non lavés étaient généralement exemptes de défauts importants. On a observé des traces d'eau libre et d'un manque d'homogénéité de coloration chez quelques beurres mais ces défauts ne se retrouvaient pas plus chez les beurres non lavés que chez les beurres lavés.

Les rapports de fabrication ont indiqué que dans quelques cas la température de barattage était si élevée que le beurre non lavé était trop tendre pour pouvoir le malaxer suffisamment. Là où l'on compte sur l'eau de lavage pour raffermir les grains de beurre en vue d'un bon malaxage, il faudra peut-être abaisșer la température de barattage pour obtenir des grains assez fermes pour bien

malaxer un beurre non lavé. Cependant, dans quelques beurreries, on a constaté par expérience que des températures de barattage plus basses que la normale ne sont pas toujours nécessaires pour obtenir une bonne fermeté et une bonne texture chez les beurres. A mesure que les beurriers ont acquis de l'expérience dans la fabrication du beurre non lavé, ils ont corrigé les petits défauts de fabrication.

Une des considérations les plus importantes pour le beurrier qui fabrique du beurre non lavé est l'augmentation de rendement due à l'accroissement de la teneur en caillé. Un surplus additionnel de $0,8 \%$ équivaut à 8.000 livres pour une fabrication totale d'un 
million de livres de beurre par année. Si l'on ne lave pas le beurre, il faut en surveiller attentivement la composition. Le beurre non lavé renfermant $15,9 \%$ d'humidité ne devrait pas recevoir plus de $2,5 \%$ de sel si le fabricant veut être sûr que la teneur en matière grasse ne soit pas inférieure à celle exigée par les rèolements. La suppression du lavage du beurre comporte d'autres avantages économiques importants. La consommation d'eau est moindre et le temps ainsi que la main-d'œuvre sont généralement diminués. La quantité d'eau résiduaire est moindre et le coût de la réfrigération peut être abaissé.

Même si l'élimination du lavage de la fabrication du beurre a certains avantages, chaque beurrerie devrait chercher à s'assurer un approvisionnement suffisant d'eau de bonne qualité qui pourra servir suivant le besoin. Les sources d'eau pour les beurreries, surtout l'eau qui vient d'un puits, devraient aussi être examinées à intervalles réguliers afin d'en déterminer la qualité bactériologique et chimique.

\section{Résumé}

On a étudié la qualité de la saveur avant et après l'entreposage ainsi que la teneur en caillé de beurres lavé et non lavé fabriqués commercialement dans 29 beurreries canadiennes. On a fait aussi des déterminations chimiques et microbiologiques.

On n'a pas noté de différence significative dans la qualité de la saveur de beurres lavés et non lavés avant et après l'entreposage.

La qualité de la saveur du beurre après six mois d'entreposage à $-5^{\circ} \mathrm{F}$. dépendait en grande partie de l'acidité initiale de la crème. Mais il n'en était pas ainsi après dix mois à $-5^{\circ} \mathrm{F}$. suivi de deux semaines à $50^{\circ} \mathrm{F}$.

La suppression du lavage a augmenté le rendement du beurre à cause de l'accroissement de la teneur en caillé (augmentation moyenne de $0,53 \%$ ).

Sous les conditions dans lesquelles l'étude a été réalisée, le lavage et l'entreposage n'ont eu aucun effet sur les valeurs chimiques utilisées pour déterminer la détérioration de la matière grasse. Le lavage a abaissé un peu la teneur en cuivre dans quelques cas mais a été sans effet sur la teneur en fer et il a relevé les valeurs $p \mathrm{H}$ de 0,2 unité. La notation de la saveur après l'entreposage n'a pas été influencée par les valeurs $p H$.

Les moyennes logarithmiques pour tous les beurres ont accusé une numération bactérienne un peu plus basse chez les beurres lavés que chez les beurres non lavés avant et après l'entreposage. Les moyennes logarithmiques des numérations bactériennes des beurres de beurreries indiriduelles ont révélé que le beurre lavé 
de 18 fabriques et le beurre non lavé de 11 avait des numérations inférieures à celles de leurs contreparties.

La notation de la saveur a été indépendante de la numération bactériennes à tous les classements pendant dix mois d'entreposage pour les deux types de beurre.

Les résultats indiquent que la suppression du lavage pendant la fabrication n'a pas contribué de façon significative à relever la teneur microbienne des beurres.

\section{Remerciements}

Nous tenons à remercier les agents de la Division des produits laitiers et de la Division de la ehimie du ministère de l'Agriculture du Canada ainsi que le personnel du Département de laiterie du Collège agricole d'Ontario pour les conseils utiles qu'ils nous ont donnés dans l'élaboration de cette étude.

Nous remercions aussi les chefs des Divisions provinciales de l'industrie laitière et leur personnel pour avoir choisi les fabriques et surveillé la fabrication du beurre. Nous sommes aussi reconnaissants envers les fabriques qui ont participé à l'étude pour la collaboration dont elles ont fait preuve.

M. C. A. Morrison du personnel de classement des produits laitiers a aidé au classement et $M$. D. C. Beckett a échantillonné les beurres après l'entreposage pour les déterminations microbiologiques. En fin, M. C. Reimer de la Section de biométrie du Service des sciences a aidé à faire l'analyse statistique des données.

\section{RÉFÉRENCES}

[1] D. K. D. Arnott, V. W. Greene and D. L. Gibson. Effect of Nonwashing on Commercial Creamery Butter. Can. Dairy Ice Cream J., $31,4: 31.1952$.

[2] J. G. Bouchard. Swett-Cream Butter. Part 11. Directions for the Manufacture of Butter from Swett or Unripened Cream. Can. Dept. Agr. Bull. 13, 1907.

[3] Canada Dairy Products Act. The Canada Dairy Products Regulations. The Canada Gazette, 88, 23, Déc. 8, 1954.

[4] Dairy Research Institute (New Zealand). 24th Ann. Rpt., 1951-52.

[5] Danish State Dairy Research Sta. 22nd, 23rd, and 24th Ann. Rpt., 1939.

[6] T. M. Jensen, W. J. Wiley, R. H. N. Trembath, and A. G. Itzerott. The Curd Content of Butter, J. Dept. Agr., Victoria, $40: 528,1942$.

[7] A. A. Lebsanft. Modified Process of Manufacturing Butter without Washing. Butterfat and Solids, 12, 3 ; 149, 1953.

[8] F. H. MoDowall, J. A. Singleton, and J. J. O'Dea. Quality and Keeping Quality of Non-washed Butter. New Zealand. J. Sci. Technol. Sec. A, $35: 175,1953$.

[9] L. E. Nichols. Dept. Agr. and. Stock, Queensland, Australia, Mimeo. Circ., 1953.

[10] M. J. O'SheA and J. Lyons. Moisture, Salt and Curd in Creamery Butter. J. Dept. Agr., Eire, 42 : 1,, 1945. 
[11] E. G. Pont. The Washing of Butter and Its Effect on Curd Content and Quality. J. Council Sci. Ind. Research, Australia, 19 : 432, 1946.

[12] E. G. Pont. The Keeping Quality of Non-Washed Butter. J. Council Sci. Ind. Research, Australia, $21: 319,1948$.

[13] R. R. Riel, A. H. White, and W. A. MoGugan. Washed and Nonwashed Butter. 11. Chemical Factors, J. Dairy Sci, , 39: 1351, 1956.

[14] A. H. White, D. M. Beattie and R. R. Riel. Washed and Nonwashed Butter. I. J. Dairy Sci., $39 ; 261,1956$.

[15] A. H. White and K. N. Smith. Washed and Non-Washed Butter 111. Microbiological Aspects. J. Dairy Sci., 39 : $1359,1956$.

[16] R. W. Wyaxt. A Study of the Incorporation of Proteins in Creamery Butter. J. Dairy Sci., 3 : 452, 1920.

\title{
DOSAGE SIMULTANÉ DU FER ET DU CUIVRE DANS LE LAIT ET LES PRODUITS LAITIERS ( 1 ) par
}

\author{
R. ALIFAX et Marie BEJAMBES
}

Station Centrale de Microbiologie et Recherches laitières, Jouy-en-Josas.

Un travail en cours sur la conservation des beurres nous a amenés à étudier les techniques de dosage de petites quantités de fer et de cuivre dans les produits laitiers. On sait en effet que leur présence a une importance considérable, ces éléments jouant un rôle catalytique prépondérant dans l'oxydation des matières grasses. Dans les laits en poudre - sauf dans ceux qui sont totalement écrémés — et dans les beurres, les altérations d'origine physico-chimique sont particulièrement redoutables. Elles s'accompagnent de l'apparition de défauts d'aspect et de goût qui diminuent considérablement la valeur marchande de ces produits et qui se manifestent surtout au cours d'une conservation prolongée. Il est donc important, avant de procéder au stockage des beurres, de vérifier si leur teneur en fer et en cuivre n'est pas telle qu'elle puisse favoriser leur altération ultérieure.

L'intérêt de cette question justifie l'abondance des travaux qu'elle a suscités. Nous n'en citerons que quelques-uns dans la suite de cet exposé.

\section{RAPPEL DES PRINCIPALES MÉTHODES DE DOSAGE DU FER ET DU CUIVRE DANS LES PRODUITS LAITIERS}

\section{A. - Préparation des échantillons}

Le dosage du fer et du cuivre dans les produits laitiers nécessite la libération préalable des cations des complexes protéiques dans

(1) Annales de Technologie agricole (I.N.R.A.), 1956, no 4, p. 619, 